\title{
Review
}

\section{Data-intensive drug development in the information age: applications of Systems Biology/Pharmacology/Toxicology}

\author{
Naoki Kiyosawa ${ }^{1}$ and Sunao Manabe ${ }^{2}$ \\ 1Translational Medicine \& Clinical Pharmacology Department, Daiichi Sankyo Co. Ltd., \\ 1-2-58 Hiromachi, Shinagawa, Tokyo 140-0005, Japan \\ ${ }^{2}$ Medical Affairs Division, Daiichi Sankyo Co. Ltd., 3-5-1 Nihonbashi Honcho, Chuo-ku, Tokyo 103-8426, Japan
}

(Received September 27, 2016)

\begin{abstract}
Pharmaceutical companies continuously face challenges to deliver new drugs with true medical value. R\&D productivity of drug development projects depends on 1) the value of the drug concept and 2) data and in-depth knowledge that are used rationally to evaluate the drug concept's validity. A model-based data-intensive drug development approach is a key competitive factor used by innovative pharmaceutical companies to reduce information bias and rationally demonstrate the value of drug concepts. Owing to the accumulation of publicly available biomedical information, our understanding of the pathophysiological mechanisms of diseases has developed considerably; it is the basis for identifying the right drug target and creating a drug concept with true medical value. Our understanding of the pathophysiological mechanisms of disease animal models can also be improved; it can thus support rational extrapolation of animal experiment results to clinical settings. The Systems Biology approach, which leverages publicly available transcriptome data, is useful for these purposes. Furthermore, applying Systems Pharmacology enables dynamic simulation of drug responses, from which key research questions to be addressed in the subsequent studies can be adequately informed. Application of Systems Biology/Pharmacology to toxicology research, namely Systems Toxicology, should considerably improve the predictability of drug-induced toxicities in clinical situations that are difficult to predict from conventional preclinical toxicology studies. Systems Biology/Pharmacology/Toxicology models can be continuously improved using iterative learn-confirm processes throughout preclinical and clinical drug discovery and development processes. Successful implementation of data-intensive drug development approaches requires cultivation of an adequate $R \& D$ culture to appreciate this approach.
\end{abstract}

Key words: Transcriptome, Toxicogenomics, Systems Biology, Systems Pharmacology, Systems Toxicology

\section{INTRODUCTION}

Considering the increased economic burden of healthcare systems in rapidly aging societies, the higher hurdle of regulatory demands, and awareness of the cost-effectiveness of the new drugs, it is crucial for sustainable growth of innovative pharmaceutical companies that R\&D productivity be improved and new drugs with true medical value, such as those that are more potent, safer, or more efficacious for patients who dissatisfied with the current standard of medications, be continuously delivered to markets. In the information age, a model-based, data-intensive drug development approach is a key competitive factor used by innovative pharmaceutical companies to mitigate uncertainties associated with undesirable information bias and rationally demonstrate the value of drug products. A concept of data-intensive drug development is presented in Fig. 1, in which two metrics to be considered for drug development are highlighted: 1) value excellence of the drug concept (i.e., why the drug should be delivered to patients), and 2) objective scientific data and in-depth knowledge to support decision-making on whether the drug concept is achievable (i.e., reliable evidence). In this manuscript, the term "data" implies all accessible internal/external information, particularly holistic and hypothesis-free-omics data (e.g., whole genome sequence, transcriptomics, proteomics) rather than "summarized" or "processed" data (e.g., targeted sequence polymorphisms

Correspondence: Naoki Kiyosawa (E-mail: kiyosawa.naoki.wr@daiichisankyo.co.jp) 


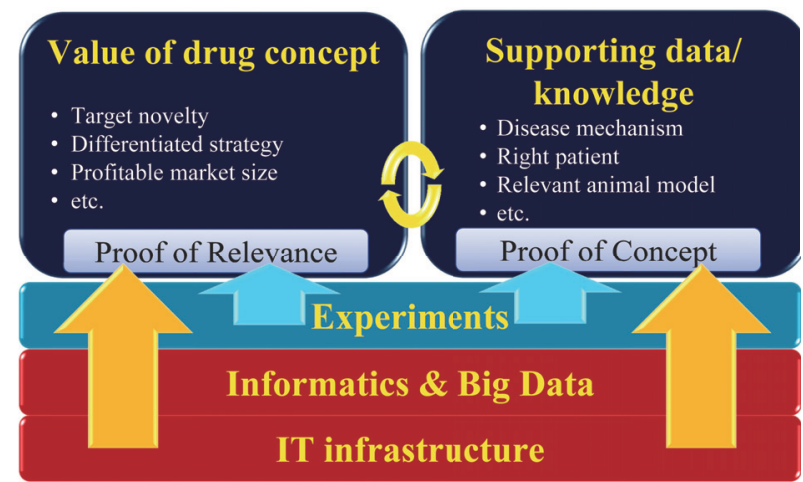

R\&D culture

Data-intensive decision-making

Model-informed drug discovery \& development

Fig. 1. Concept of data-intensive drug development. The R\&D productivity of drug development projects depends on 1) the value excellence of the drug concept and 2) supporting data and in-depth knowledge to demonstrate the validity of the drug concept. In the information age, a vast amount of biomedical information can be publicly available, and researchers can obtain relevant information by using informatics techniques along with preclinical and clinical experiments. Furthermore, in addition to adequate investment on establishing infrastructures to support informatics analysis, cultivation of an adequate $R \& D$ culture to acknowledge the value of a data-intensive approach will be important for the successful implementation of such an approach.

data), personal experience, preference, or opinions from authorities, all of which are inevitably not free from certain information biases. A data-intensive approach aims to develop rational models based on all accessible data that should be continuously improved by incorporating novel information obtained either externally or internally. These models can be used to support planning of rational study designs and to improve decision-making by using a quantitative approach.

The value of "Big Data" is defined as "high-volume, high-velocity, and high-variety information assets that demand cost-effective, innovative forms of information processing for enhanced insight and decision making" (Yin and Kaynak, 2015) and is attracting more social interest, so scientists are also becoming more aware of its potential to strengthen mechanistic understandings of both disease pathophysiology and drug actions in animals. In this manuscript, case examples of Systems Biology/Pharmacology applications will be presented as examples of data-intensive translational research in the drug development process. Application of Systems Biology/Pharma- cology to toxicology research, namely Systems Toxicology, will also be presented.

\section{Public transcriptome data as a valuable source of biomedical Big Data}

Although there is a vast amount of biomedical information in the public domain, such as genome sequence, epigenome, transcriptome, chemicals, and biological pathways (Ma'ayan et al., 2014), most of the public data is not ready for straightforward computation because of their unstructured and diverse formats and the extremely large amount of the data, which is growing daily. In addition, there are a number of challenging factors in the handling of Big Data, such as insufficient annotation, batch differences, technical variability, and platform differences (Khan et al., 2014), all of which make computation challenging. Among the varieties of biomedical information, transcriptome data is generally well structured and relatively convenient for computation, and the amount of publicly available transcriptome data has increased dramatically and is continuing to grow in an exponential manner (Fig. 2). Representative public transcriptome databases include the Gene Expression Omnibus (Barrett et al., 2013) and ArrayExpress (Rustici et al., 2013).

Nevertheless, handling large-scale transcriptome data is still technically difficult for ordinary biologists, who are unfamiliar with informatics analysis (i.e., mathematical and computations knowledge/skills). In addition, the analyzed results tend to be too descriptive and complicated for biologists to draw actionable conclusions. To address such difficulties, a gene set-level analysis approach instead of individual mRNA level analysis appears to work well, reducing the complexity of the transcriptome data and providing comprehensive systems-level functional profiles (e.g., pathway-level activation rather than an increase in the mRNA of individual genes) or certain toxicological endpoints (Kiyosawa et al., 2010; Bureeva and Nikolsky, 2011). To implement such gene set-level analysis, a sufficient number of high-quality gene sets needs to be prepared in advance for which public knowledgebases, such as Gene Ontology (Ashburner et al., 2000), DAVID (Huang da et al., 2009), MSigDB (Liberzon et al., 2011), or GeneSetDB (Araki et al., 2012), can be a useful resource, although the gene sets retrieved from these knowledgebases might not be straightforwardly optimal for transcriptome profiling use. On the other hand, accumulation of publicly available transcriptome data supports generation of high-quality gene sets optimal for evaluating transcriptome data, which leads to development of a proprietary knowledgebase to support mechanistic interpretation of complicated transcriptome data on 
Data-intensive Systems Biology/Toxicology/Pharmacology approaches

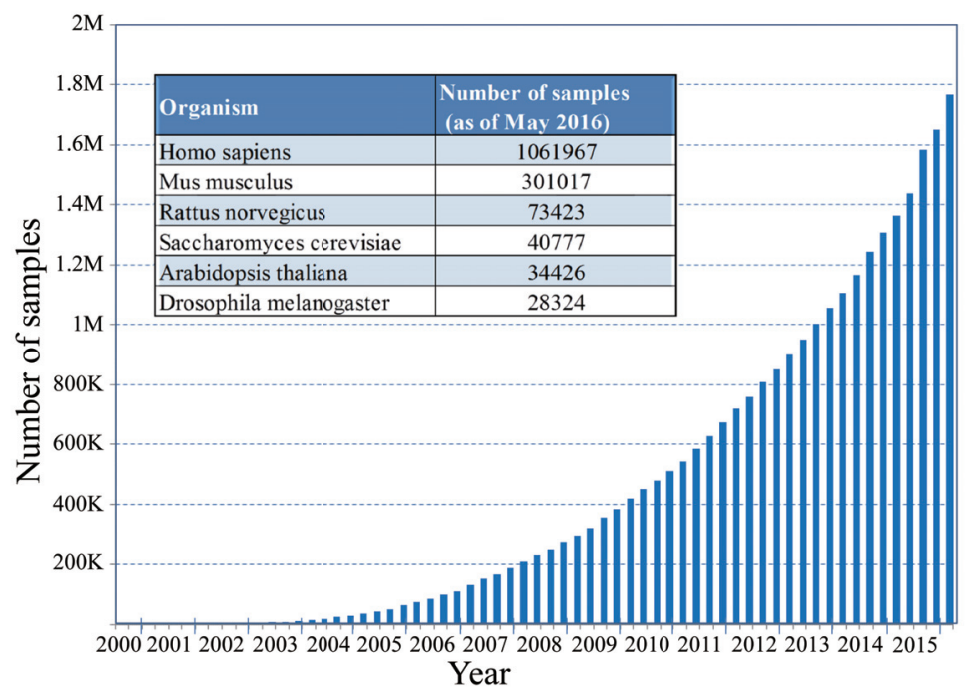

Fig. 2. Accumulation of transcriptome data in the Gene Expression Omnibus (GEO) database. The amount of transcriptome data (e.g., microarray or RNA-Seq) continues to increase in an exponential manner in publicly available transcriptome databases, such as the Gene Expression Omnibus (GEO; http://www.ncbi.nlm.nih.gov/geo/).

disease, animal model pathophysiology, or drug-induced toxicity (Fig. 3).

\section{Disease and animal model profiling to promote understanding of pathophysiological mechanisms}

Mechanistic profiling using patient transcriptome data, or disease profiling analysis, can be performed any time, provided adequate patient transcriptome data and gene set information are available. Fig. 4 represents a case example of disease profiling analysis of synovial tissue from patients with rheumatoid arthritis (GEO accession number: GSE48780), which indicates the existence of mechanistic heterogeneity among patients; e.g., some exhibit stimulation of TNF, IL1A, IL8, IFNG, and NFkB (Fig. 4A), whereas others exhibit stimulation of IL4 and FGF (Fig. 4B). These results imply that we might need to consider right target patients depending on the mechanisms of actions of the drugs. For such disease profiling analysis, the more gene sets we prepare, the higher the resolution of mechanistic profiling results that can be obtained. It is ideal to perform disease profiling at the earliest opportunity in the drug development process considering that a detailed understanding of a disease is the basis of creating right drug concept (i.e., right drug target and right patient population), creating adequate study designs, and preparing for fit-for-purpose biomarker measurement systems in the clinical studies.

Disease profiling analysis can be performed not only for humans but also for experimental animal models. In many cases, selection of animal models used for pharmacology studies tends to be based on phenotypic similarities to those of patients, which does not always guaran-

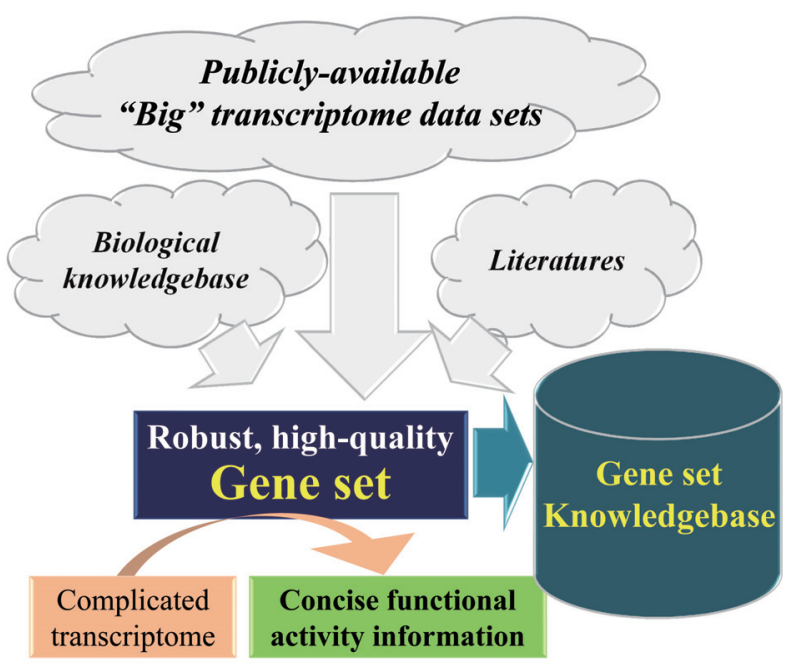

Fig. 3. Preparation of gene set knowledgebase. Gene sets with expression levels that are closely associated with certain biological pathways or toxicological endpoints can be a valuable knowledgebase for characterizing the functional status of biological systems using complicated transcriptome data, which can be applied to disease profiling (Fig. 4) or animal model profiling (Fig. 5). 


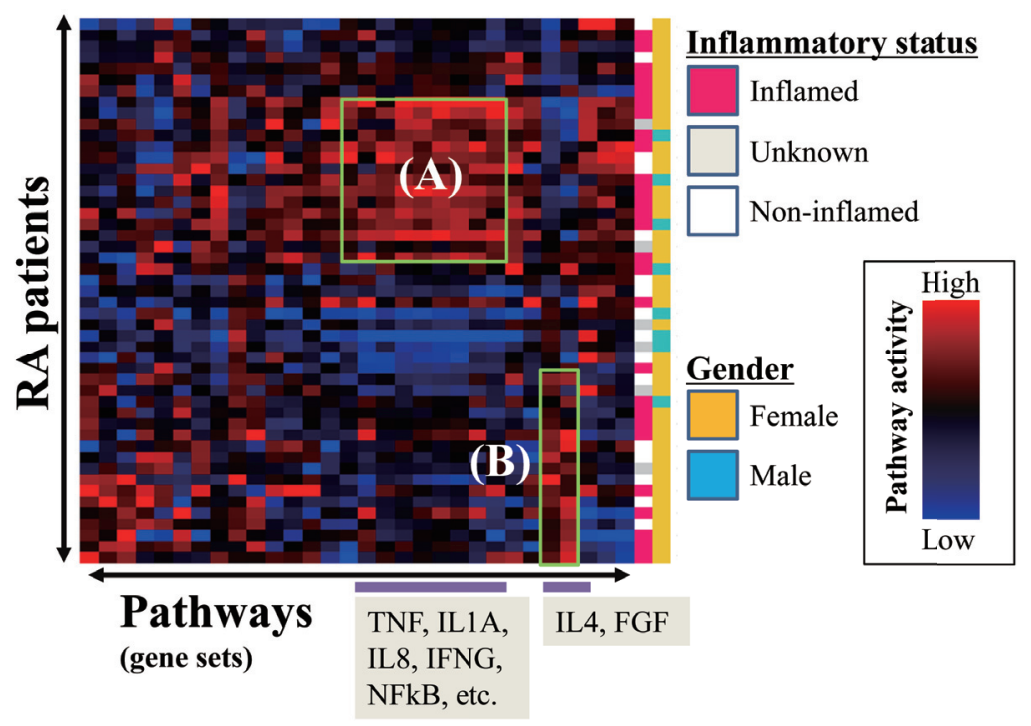

Fig. 4. Disease profiling to promote understanding of diseases. A case example of disease profiling in synovial tissues from patients with rheumatoid arthritis using transcriptome data (GEO accession number GSE48780) is presented. The results show clear mechanistic heterogeneity among patients. (A) Subjects with activations of TNF, IL1A, IL8, IFNG, and NFkB (B) Subjects with activations of IL4 and FGF. In the disease profiling analysis, the greater the number of gene sets, the higher the resolution of mechanistic profiling that can be achieved.

tee mechanistic relevance to patients' pathophysiological mechanisms. Accordingly, it is unsurprising that extrapolating preclinical study results to humans is not always satisfactory. Figure 5 presents a comparison of the pathophysiological mechanisms between psoriasis patients (GSE14905) and animal disease models exhibiting skin lesion phenotypes (GSE27628 and GSE50400), which indicates that both K14-AREG transgenic mice and IL-23 -injected mice exhibited marked activations of IFNA and IL-17 similar to the case in psoriasis patients, whereas imiquimod-induced mice did not exhibit activation of IFNA. Accordingly, when focusing on IFNA and IL-17 as hallmarks of psoriasis pathology, K14-AREG transgenic mice and IL-23-injected mice may be more relevant to psoriatic patients than are imiquimod-treated mice. It should be noted that animal model profiling results might vary depending on differences in experimental conditions (e.g., animal strain, time point of observation, drug dose level). Ideally, such mechanistic profiling should be performed in every key animal experiment in order to support adequate interpretation of the study results.

\section{Network modeling to explore key driver pathways underlying pathophysiology}

Although disease profiling (Fig. 4) and animal model profiling (Fig. 5) can facilitate comprehension of sys- tems-level functional profiles in both patients and animals, the results do not explicitly identify "key driver pathways" underlying the pathophysiological conditions. To decipher key driver pathways in the complicated biological systems, a network modeling technique is one of the practical options. Briefly, the initial step to develop a network model is to determine differentially expressed genes (DEGs) in the pathophysiological condition (e.g., patients or animal models). DEGs are then subjected to pathway enrichment analysis to extract a set of biological pathways that over-represent the DEG genes, which are eventually integrated into a single network model that highlights key pathophysiological mechanisms in the patients or animal models. To implement the pathway enrichment analysis, a high-quality and sufficiently complete reference pathway knowledgebase is required, such as the KEGG (Kanehisa et al., 2016) or Reactome (Fabregat et al., 2016) in the public domain, or commercially provided resource, such as Ingenuity ${ }^{\circledR}$ Pathway Analysis (Qiagen, Venlo, Netherlands) and MetaCore ${ }^{\mathrm{TM}}$ (Thomson Reuters, New York, NY, USA). Figure 6 represents a network model of psoriasis based on patient skin transcriptome data (GSE14905), in which disease-profiling results are adequately incorporated to highlight functionally active pathways and remove less-affected pathways. The psoriasis network model in Fig. 6 demonstrates 
(A)

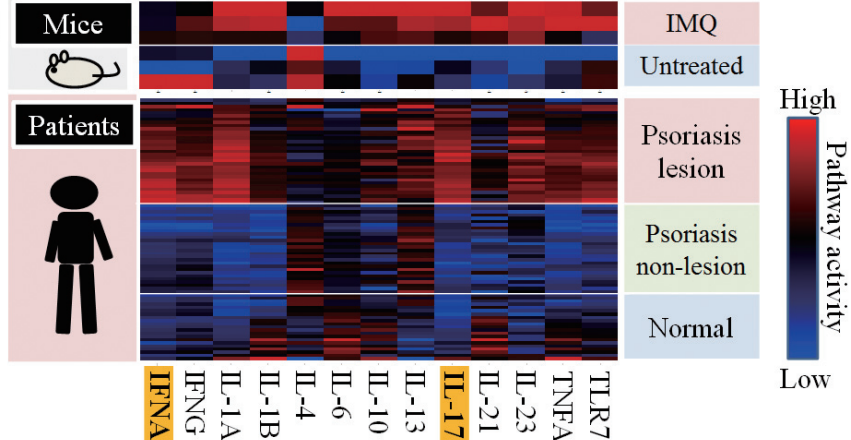

(B)

\begin{tabular}{|c|c|c|c|c|}
\multirow{2}{*}{ Pathway } & \multicolumn{3}{|c|}{ Animal model } & \multirow{2}{*}{$\begin{array}{c}\text { Psoriasis } \\
\text { patient }\end{array}$} \\
\cline { 2 - 5 } & IMQ model & K14-AREG Tg & IL-23 model & $\uparrow$ \\
\hline IFNA & No change & $\uparrow$ & $\uparrow$ & $\uparrow$ \\
\hline IL-17 & $\uparrow$ & $\uparrow$ & $\uparrow$ & $\uparrow$ \\
\hline
\end{tabular}

Fig. 5. Animal model profiling to evaluate mechanistic relevance to patients. (A) An example of comparing mechanistic profiles between skin biopsies from patients with psoriasis (GSE14905) and imiquimod (IMQ)-induced skin lesion model mice (GSE60804). IMQ is reported to act as an agonist to TLR7. (B) Functional profiles of hallmark disease pathways for patients with psoriasis and corresponding animal disease models with skin lesions (GSE27628 and GSE50400). The results indicate that both K14-AREG transgenic mice and IL-23-injected mice exhibited marked activations of IFNA and IL-17 signals similar to those of patients with psoriasis, whereas IMQ-induced mice did not exhibit activation of IFNA, thus suggesting that both K14-AREG transgenic mice and IL-23-injected mice, rather than IMQ-treated mice, might be more relevant to patients with psoriasis when focusing on IFNA and IL-17.

that the IL-23-IL-17 axis appeared to be a hub element directly affecting three major inflammatory pathways (i.e., JAK, ERK, and NFkB), which suggests its role as a key driver in regulation of the inflammatory pathophysiology in the skin of patients with psoriasis, which has now been well demonstrated (Di Cesare et al., 2009). Thus, the network modeling technique can add value to disease profiling results for investigating potential key driver pathways in a pathophysiological condition and support selection of right drug target, which is the basis for successful drug development (Cook et al., 2014).

One of the major limitations of the network modeling technique is that it provides only qualitative information (i.e., relationships among genes or pathways) and does not enable dynamic simulation to evaluate drug responses, for which application of Modeling \& Simulation (M\&S) techniques will be needed. The following sections briefly discuss applications of 1) Systems Pharmacology and 2) Pharmacometrics as two method subsets of M\&S according to the classification used in a previous report (Marshall et al., 2016).

\section{Modeling \& Simulation: Systems Pharmacology and Pharmacometrics}

Systems Pharmacology is a discipline used to develop mathematical models that describe a drug exposureresponse relationship by combining a top-down datadriven approach and bottom-up, theory-driven, Systems Biology-based mechanistic details to support the design of therapeutic interventions (Vicini and van der Graaf, 2013), for which mechanistic detail can be at the level of both/either pharmacokinetic (PK) and/or drug responses. As discussed previously, Systems Biology analysis (e.g., disease profiling, animal model profiling and network modeling) can provide the basis of pathophysiological mechanisms that potentially affect drug responses. In case the mechanistic relevance of animal models to patients remains questionable because of uncertainties (such as in the pathophysiological mechanisms), anatomical differences in target organs, or potential inter-subject variability in patients, one practical option is to develop a Quantitative Systems Pharmacology (QSP) model, in which all relevant internal/external information is incorporated (e.g., disease pathophysiology, clinical data for competitor drugs, in vitro pharmacology data, etc.). For instance, prediction of drug responses in patients with central nerv- 


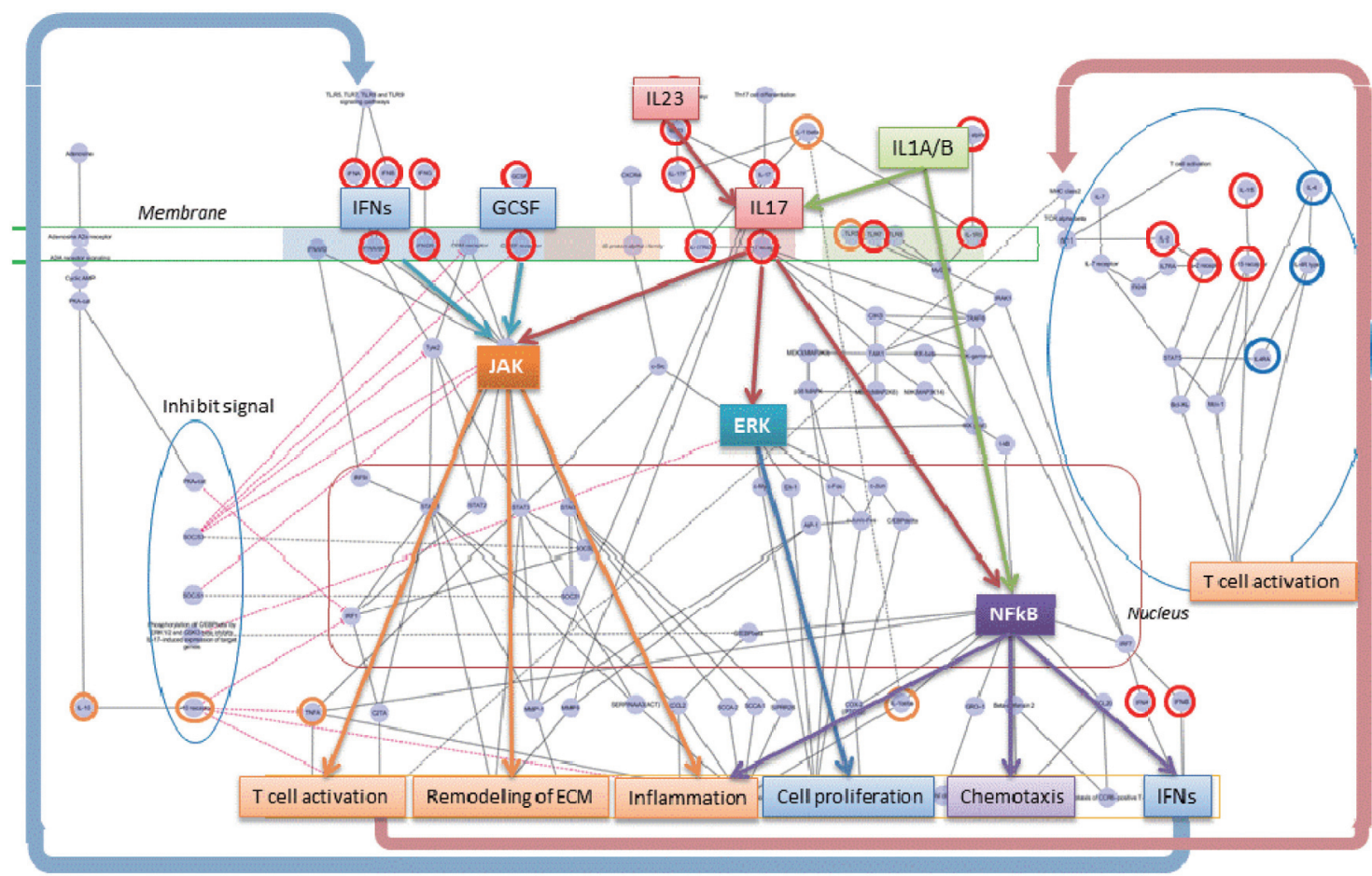

Fig. 6. Network modeling to highlight key driver pathways. A network model was developed by using transcriptome data of patients with psoriasis skins (GSE14905), supported by pathway knowledgebase and disease-profiling results to highlight functionally active pathways. The network model demonstrates IL-23 and IL-17 as hub elements affecting three major inflammatory pathways (i.e., JAK, ERK, and NFkB) in the network model, which suggests their roles as potential key drivers of inflammatory pathophysiology of the skin of patients with psoriasis.

ous system disorders by experiments with animal models is extremely challenging in general, and applications of QSP are expected to improve prediction of clinical drug responses (Geerts et al., 2012, 2013). It should be noted that the QSP model might not necessarily need to represent the completely "true" biological/pharmacological mechanisms; instead, it would be more appropriate to regard the QSP model as a knowledgebase, in which our best knowledge and hypothesis at present are integrated, and the QSP model is continuously updated by incorporating novel information (e.g., new scientific discoveries and both positive/negative study results against model prediction). In addition, researchers will identify key research questions and uncertainties to be addressed in the preclinical and clinical studies during the modeling process, which is another valuable advantage of developing a QSP model. In other words, QSP might not necessarily be used to "forecast" the clinical outcomes of a drug treatment, but rather to "inform" scientists as to which information is missing or uncertain and should be addressed in upcoming adequately designed studies.

Pharmacometrics is a discipline used to develop math- ematical models to characterize, explain, and predict PK and drug responses, combined with statistical models to quantify the source and extent of variability and uncertainty in the underlying process (Marshall et al., 2016). All of the drug response data (e.g., efficacy, safety and any relevant biomarkers) collected through the entire drug development process will be eventually subjected to Pharmacometrics modeling quantitatively to characterize the drug exposure-response relationship, which is essential for demonstrating how the drug affects the body and for optimization of the dosage regimen.

\section{Systems Toxicology: applications of the data-intensive approach to toxicology}

Application of the data-intensive approach is valuable, not only for evaluating drug efficacy but also for improving the quality of toxicology research. Currently, preclinical drug safety assessment depends largely on empirical and descriptive approaches using healthy animals. Although descriptive toxicology approaches using animals can well predict human toxicities in general (Olson et al., 2000), it is not rare to observe severe drug-induced 
Data-intensive Systems Biology/Toxicology/Pharmacology approaches

toxicities, such as clinical idiosyncratic drug-induced liver injury (Kaplowitz, 2005), which occurs with few signs of toxicity potential in the animal studies. Difficulty in the translatability of preclinical findings to clinical situations is attributed to insufficient understanding of species differences in pathophysiological mechanisms and drug actions, for which the Systems Toxicology approach should be of great help, providing further mechanistic insights.

As an early attempt at applying the Systems Toxicology approach, pharmaceutical companies embarked on toxicogenomics research in the late 1990 s to early 2000 s, when generation of transcriptome data was very costly and researchers were unwilling to share their valuable data in the public domain. As a pioneering challenge in Japan, a precompetitive consortium by government, academia, and pharmaceutical companies, called the Toxicogenomics Project in Japan, was initiated in 2003 to construct a high-quality, large-scale, toxicogenomics database called TG-GATEs for gathering transcriptome data of rat liver and kidney and of hepatocytes of rats and humans treated with 170 drugs (Uehara et al., 2010; Igarashi et al., 2015). Now, there are a number of toxicogenomics databases in the public domain, such as DrugMatrix (Ganter et al., 2005) and Chemical Effects in Biological Systems (Waters et al., 2008). There are also many toxicogenomic data sets in the GEO or ArrayExpress databases, all of which provide an excellent resource for toxicology researchers in the modern information age.

Regarding gene set knowledge to support interpretation of toxicogenomics data, a large amount of gene set information has been reported for evaluating certain toxicities, such as drug-induced glutathione depletion (Kiyosawa et al., 2007) and carcinogenicity (Uehara et al., 2008). Such gene sets can be used either to develop predictive models (Uehara et al., 2008), analyze functional enrichment (Subramanian et al., 2005), or evaluate functional activities of toxicological endpoints (Martin et al., 2012; Kiyosawa et al., 2009). Recent research trends suggest that application of toxicogenomics to biomarker discovery and development has diminished, whereas application to mechanistic studies remains active (Chen et al., 2012). An application example of toxicogenomics profiling (or "drug profiling") can be found in our previous report (Kiyosawa et al., 2010), in which the time course of functional activities in various biological pathways and toxicological endpoints following bromobenzene treatment could be concisely summarized by using pre-defined toxicogenomic gene sets, which provided mechanistic insights into liver toxicity.

The mechanistic effects following drug treatments vary depending on the mechanistic conditions in the body. Disease model animals may be useful not only for evaluating drug efficacy but also drug safety. For example, doselimiting pharmacologic effects of glucose-lowering drugs have been observed in non-diseased animal models (i.e., animals used in ordinal toxicology studies) at relatively low multiples of therapeutic exposure, which may lead to overestimation of the hypoglycemic risk in patients with diabetes (Morgan et al., 2013). Thus, interpretation of the preclinical toxicology study results on the basis of a profound understanding of disease model animals may be improved for predicting clinical safety in certain cases.

Regarding technical aspects of transcriptome data collection, although microarray has been a major platform for collection of transcriptome data, the RNA-Seq technique using Next Generation Sequencing technology is now increasingly being used because of its comprehensiveness, sensitivity, and additive information, such as alternative splicing and non-coding RNAs. Fortunately, it has been suggested that gene set-level knowledge derived from microarray experiments can be useful for RNA-Seq data, which secures the value of legacy microarray data and gene set knowledge in the RNA-Seq era (Xu et al., 2016; Wang et al., 2014; Su et al., 2014). In addition, RNA-Seq technology can generate reasonable quality of transcriptome data from formalin-fixed, paraffin-embedded, (FFPE) tissue samples, and the comparability of the FFPE-derived transcriptome profile to that from fresh samples can be improved by conducting gene set-level analysis instead of individual gene-level analysis (Webster et al., 2015). Accordingly, FFPE samples collected in legacy toxicology studies could now be a valuable resource for investigation of mechanisms of toxicities observed in long-term studies, such as rodent carcinogenesis studies.

Regarding Systems Pharmacology applications to toxicology, a promising example can be found in the case of the DILIsym ${ }^{\mathrm{TM}}$ model, which focuses on specific hepatotoxicity-related mechanisms, such as glutathione depletion, mitochondrial dysfunction, or drug ADME, and has enabled simulation of the drug-induced hepatotoxic potential in humans by using preclinical in vitro/in vivo data (Howell et al., 2012). By setting a variety of adequate parameter sets in the DILIsym model to represent a healthy human population (i.e., virtual human subjects), the simulation may indicate rare but serious drug-induced hepatotoxic potential effects that are difficult to predict by using conventional preclinical toxicology studies in animals (Yang et al., 2014).

Collectively, although the conditions and mechanisms underlying drug-induced toxicities are generally far more 
complicated than those of simple pharmacology actions of drugs (e.g., higher drug exposure, effects from tissue damage or endocrine systems other than direct pharmacological effects, and disruption of cellular homeostasis), data-intensive approaches should still apply to toxicology research and will considerably improve the predictability of clinical drug-induced toxicities by adding mechanistic and quantitative insights into general toxicity profiles with key safety parameters (e.g., MTD, NOAEL, and HNSTD) in animals, as discussed in a previous publication (Sahota et al., 2016).

\section{Summary and future perspective}

Figure 7 summarizes a representative analytical flow of a data-intensive approach combining Systems Biology/ Toxicology/Pharmacology techniques, starting from transcriptome data (e.g., patient or animal models) and gene set knowledgebase. Systems Biology/Toxicology analysis will contribute toward 1) a profound understanding of pathophysiological mechanisms of disease (or toxicity), which is the basis for creating the right drug concept with true medical value, 2) interpreting preclinical study results through a profound understanding of the pathophysiology of animal models, and 3) highlighting potential key disease driver pathways to be intensely investigated. During the preclinical development stage, along with gold-standard animal model studies, application of QSP may improve the predictability of clinical drug responses. Moreover, QSP can guide researchers in preparing an adequate study design. All collected data obtained through the entire drug development process will be subject to modeling of the drug exposure-response relationship by using pharmacometrics techniques, which is an essential approach for demonstrating drug effects and optimizing the drug's dose regimen. Decision making and study planning should be adequately guided by fit-for-purpose models based on the best biological and pharmacological knowledge, and the models should be continuously updated and refined during the drug development process in alignment with the concept of the learn-confirm process (Lalonde et al., 2007). The best practice using "Model-informed drug discovery \& development (MID3)"

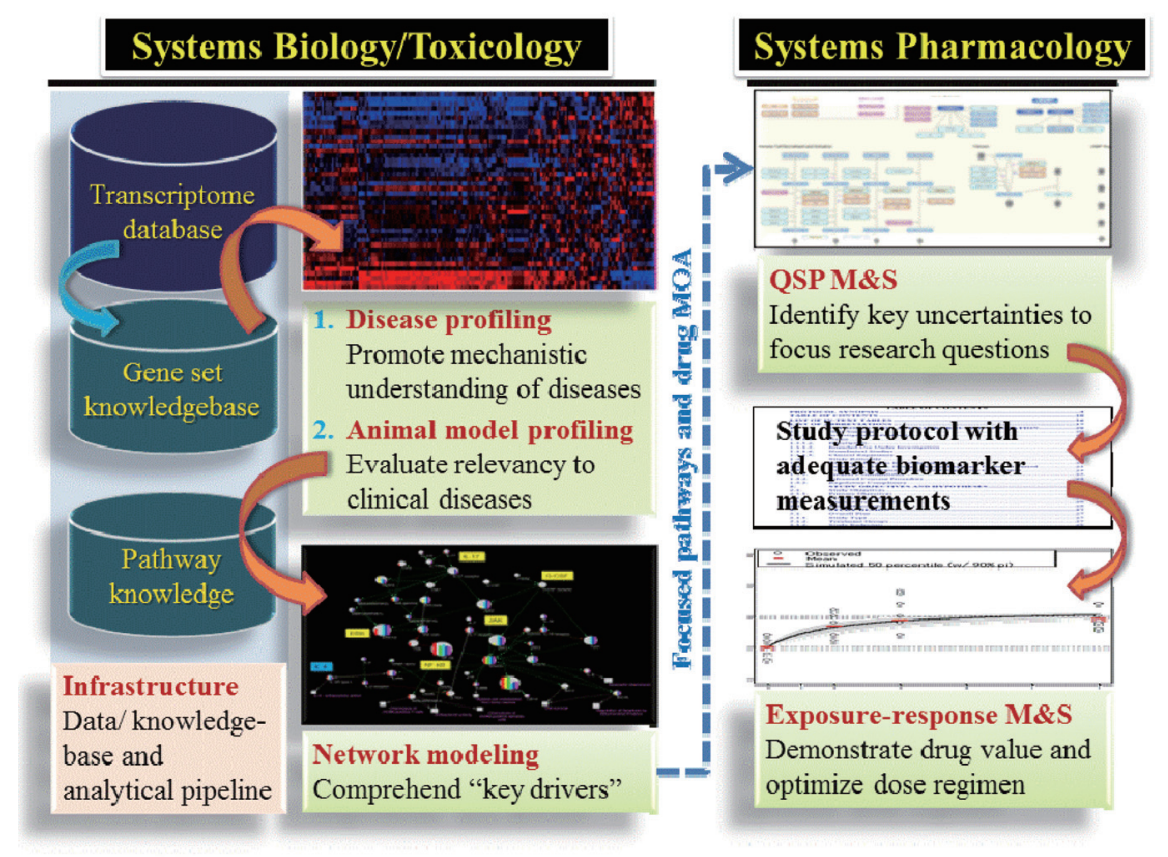

Fig. 7. Analytical flow of Systems Biology/Pharmacology/Toxicology. A representative analytical flow of Systems Biology/Pharmacology/Toxicology is presented. Mechanisms of pathophysiology/toxicity are investigated by using patient transcriptome data and gene set knowledgebase (disease profiling). Network modeling supported by using a biological pathway knowledgebase can be developed from patient transcriptome data, which can highlight key disease pathways. A quantitative Systems Pharmacology model is developed that is based on focused pathways related to disease and drug action, which simulate clinical responses to drug treatment and provide key research questions to be addressed in subsequent studies. All relevant data obtained through the entire drug development process are eventually subjected to the modeling exposureresponse relationship by using the Pharmacometrics technique to demonstrate the value of the drug and to optimize the dose regimen. 
Data-intensive Systems Biology/Toxicology/Pharmacology approaches

was recently introduced (Marshall et al., 2016), in which MID3 is defined as "a holistic term to characterize a variety of quantitative approaches used to improve the quality, efficiency, and cost-effectiveness of decision making through "fit-for-purpose" data analysis and prediction." Indeed, the analytical flow depicted in Fig. 7 is a practical example of MID3. Figure 8 presents a more generalized scheme of the data-intensive drug discovery and development process. For successful implementation of data-intensive drug development, not only financial investment in the computational infrastructure (e.g., data, databases, and analytical tools with cutting-edge algorithms) but also cultivation of an adequate R\&D culture is essential, as shown in Fig. 1.

Finally, information technology is evolving continuously and rapidly. For example, application of cognitive computing technology has already been initiated for discovery of biological knowledge and drug repurposing (Chen et al., 2016) and cancer diagnostics; its performance will likely evolve through the continuous acquisition of more biomedical information. On the other hand, cutting-edge neural network algorithms (i.e., deep learning) may potentially revolutionize the drug discovery process (Mamoshina et al., 2016), application of which is likely to also benefit evaluation of drug-induced toxicity (Xu et al., 2015). Thus, pharmaceutical companies will inevitably need to transform their entire drug development processes in the near future to adapt to truly data-driven drug discovery and development and continually deliver innovative drugs to patients in need.

\section{ACKNOWLEDGMENTS}

The authors are grateful to Drs. Taro Tokui and Taketoshi Ogawa for their strong leadership in promoting Translational Research Initiatives in Daiichi Sankyo and for reviewing the manuscript. The authors acknowledge contributions to Systems Biology research activities by Drs. Kenji Watanabe, Shinnosuke Yamada, Masunobu Sugimura, and Yoshiyuki Hizukuri and to Toxicogenomics research activities by Drs. Takashi Yamoto, Kazumi Ito, Kazunori Fujimoto, Kyoko Watanabe, Noriyo Niino, Shusuke Yamauchi, and Takuma Iguchi. The authors also acknowledge Dr. Kazutaka Yoshihara for reviewing the manuscript.

Conflict of interest---- The authors declare that there is no conflict of interest.

\section{REFERENCES}

Araki, H., Knapp, C., Tsai, P. and Print, C. (2012): GeneSetDB: A comprehensive meta-database, statistical and visualisation framework for gene set analysis. FEBS Open Bio, 2, 76-82.

Ashburner, M., Ball, C.A., Blake, J.A., Botstein, D., Butler, H., Cherry, J.M., Davis, A.P., Dolinski, K., Dwight, S.S., Eppig, J.T., Harris, M.A., Hill, D.P., Issel-Tarver, L., Kasarskis, A.,

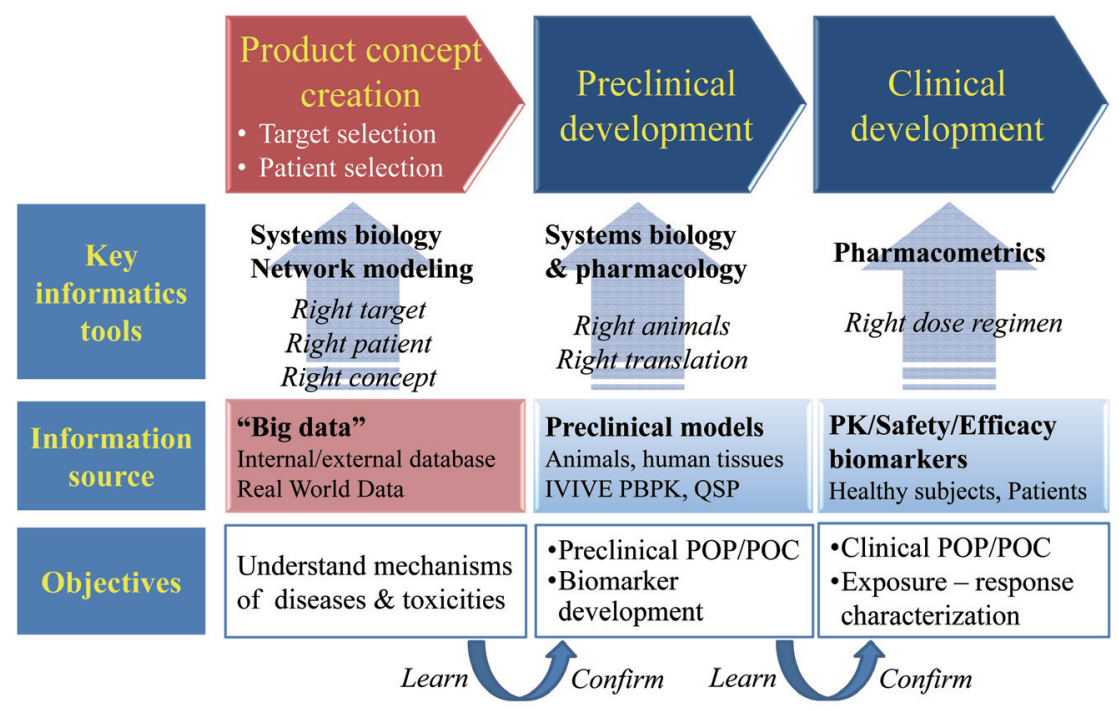

Fig. 8. Drug development process by using data-intensive approaches. A profound understanding of pathology and toxicity in humans and animal models is the fundamental basis for creating a drug concept with true medical value. The value of the drug needs to be eventually demonstrated by obtaining objective evidence throughout the entire R\&D process, for which data-intensive approaches should adequately guide researchers in creating optimal study designs and in rational decision making. 
Lewis, S., Matese, J.C., Richardson, J.E., Ringwald, M., Rubin, G.M. and Sherlock, G. (2000): Gene ontology: tool for the unification of biology. The Gene Ontology Consortium. Nat. Genet., 25, 25-29.

Barrett, T., Wilhite, S.E., Ledoux, P., Evangelista, C., Kim, I.F., Tomashevsky, M., Marshall, K.A., Phillippy, K.H., Sherman, P.M., Holko, M., Yefanov, A., Lee, H., Zhang, N., Robertson, C.L., Serova, N., Davis, S. and Soboleva, A. (2013): NCBI GEO: archive for functional genomics data sets-update. Nucleic Acids Res., 41, D991-995.

Bureeva, S. and Nikolsky, Y. (2011): Quantitative knowledge-based analysis in compound safety assessment. Expert Opin. Drug Metab. Toxicol., 7, 287-298.

Chen, M., Zhang, M., Borlak, J. and Tong, W. (2012): A decade of toxicogenomic research and its contribution to toxicological science. Toxicol. Sci., 130, 217-228.

Chen, Y., Elenee Argentinis, J.D. and Weber, G. (2016): IBM Watson: How Cognitive Computing Can Be Applied to Big Data Challenges in Life Sciences Research. Clin. Ther., 38, 688-701.

Cook, D., Brown, D., Alexander, R., March, R., Morgan, P., Satterthwaite, G. and Pangalos, M.N. (2014): Lessons learned from the fate of AstraZeneca's drug pipeline: a five-dimensional framework. Nat. Rev. Drug. Discov., 13, 419-431.

Di Cesare, A., Di Meglio, P. and Nestle, F.O. (2009): The IL-23/ Th17 axis in the immunopathogenesis of psoriasis. J. Invest. Dermatol., 129, 1339-1350.

Fabregat, A., Sidiropoulos, K., Garapati, P., Gillespie, M., Hausmann, K., Haw, R., Jassal, B., Jupe, S., Korninger, F., McKay, S., Matthews, L., May, B., Milacic, M., Rothfels, K., Shamovsky, V., Webber, M., Weiser, J., Williams, M., Wu, G., Stein, L., Hermjakob, H. and D'Eustachio, P. (2016): The Reactome pathway Knowledgebase. Nucleic Acids Res., 44, D481487.

Ganter, B., Tugendreich, S., Pearson, C.I., Ayanoglu, E., Baumhueter, S., Bostian, K.A., Brady, L., Browne, L.J., Calvin, J.T., Day, G.J., Breckenridge, N., Dunlea, S., Eynon, B.P., Furness, L.M., Ferng, J., Fielden, M.R., Fujimoto, S.Y., Gong, L., Hu, C., Idury, R., Judo, M.S., Kolaja, K.L., Lee, M.D., McSorley, C., Minor, J.M., Nair, R.V., Natsoulis, G., Nguyen, P., Nicholson, S.M., Pham, H., Roter, A.H., Sun, D., Tan, S., Thode, S., Tolley, A.M., Vladimirova, A., Yang, J., Zhou, Z., Jarnagin, K. (2005): Development of a large-scale chemogenomics database to improve drug candidate selection and to understand mechanisms of chemical toxicity and action. J. Biotechnol., 119, 219-244.

Geerts, H., Spiros, A., Roberts, P. and Carr, R. (2012): Has the time come for predictive computer modeling in CNS drug discovery and development? CPT Pharmacometrics Syst. Pharmacol., 1, e16.

Geerts, H., Spiros, A., Roberts, P. and Carr, R. (2013): Quantitative systems pharmacology as an extension of PK/PD modeling in CNS research and development. J. Pharmacokinet. Pharmacodyn., 40, 257-265.

Howell, B.A., Yang, Y., Kumar, R., Woodhead, J.L., Harrill, A.H., Clewell, H.J. $3^{\text {rd }}$., Andersen, M.E., Siler, S.Q. and Watkins, P.B. (2012): In vitro to in vivo extrapolation and species response comparisons for drug-induced liver injury (DILI) using DILIsym $^{\mathrm{TM}}$ : a mechanistic, mathematical model of DILI. J. Pharmacokinet. Pharmacodyn., 39, 527-541.

Huang da, W., Sherman, B.T. and Lempicki, R.A. (2009): Systematic and integrative analysis of large gene lists using DAVID bioinformatics resources. Nat. Protoc., 4, 44-57.

Igarashi, Y., Nakatsu, N., Yamashita, T., Ono, A., Ohno, Y.,
Urushidani, T. and Yamada, H. (2015): Open TG-GATEs: a large-scale toxicogenomics database. Nucleic Acids Res., 43, D921-927.

Kanehisa, M., Sato, Y., Kawashima, M., Furumichi, M. and Tanabe, M. (2016): KEGG as a reference resource for gene and protein annotation. Nucleic Acids Res., 44, D457-462.

Kaplowitz, N. (2005): Idiosyncratic drug hepatotoxicity. Nat. Rev. Drug Discov., 4, 489-499.

Khan, N. Yaqoob, I., Hashem, I.A., Inayat, Z., Ali, W.K., Alam, M., Shiraz, M. and Gani, A. (2014): Big data: survey, technologies, opportunities, and challenges. Sci. World J., 2014, 712826.

Kiyosawa, N., Ando, Y., Watanabe, K., Niino, N., Manabe, S. and Yamoto, T. (2009): Scoring multiple toxicological endpoints using a toxicogenomic database. Toxicol. Lett., 188, 91-97.

Kiyosawa, N., Manabe, S., Yamoto, T. and Sanbuissho, A. (2010): Practical application of toxicogenomics for profiling toxicant-induced biological perturbations. Int. J. Mol. Sci., 11, 3397-3412.

Kiyosawa, N., Uehara, T., Gao, W., Omura, K., Hirode, M., Shimizu, T., Mizukawa, Y., Ono, A., Miyagishima, T., Nagao, T. and Urushidani, T. (2007): Identification of glutathione depletion-responsive genes using phorone-treated rat liver. J. Toxicol. Sci., 32, 469-486.

Lalonde, R.L., Kowalski, K.G., Hutmacher, M.M., Ewy, W., Nichols, D.J., Milligan, P.A., Corrigan, B.W., Lockwood, P.A., Marshall, S.A., Benincosa, L.J., Tensfeldt, T.G., Parivar, K., Amantea, M., Glue, P., Koide, H. and Miller, R. (2007): Modelbased drug development. Clin. Pharmacol. Ther., 82, 21-32.

Liberzon, A., Subramanian, A., Pinchback, R., Thorvaldsdottir, H., Tamayo, P. and Mesirov, J.P. (2011): Molecular signatures database (MSigDB) 3.0. Bioinformatics, 27, 1739-1740.

Ma'ayan, A., Rouillard, A.D., Clark, N.R., Wang, Z., Duan, Q. and Kou, Y. (2014): Lean Big Data integration in systems biology and systems pharmacology. Trends Pharmacol. Sci., 35, 450460.

Mamoshina, P., Vieira, A., Putin, E. and Zhavoronkov, A. (2016): Applications of Deep Learning in Biomedicine. Mol. Pharm., 13, $1445-1454$

EFPIA MID3 Workgroup, Marshall, S.F., Burghaus, R., Cosson, V., Cheung, S.Y., Chenel, M., DellaPasqua, O., Frey, N., Hamrén, B., Harnisch, L., Ivanow, F., Kerbusch, T., Lippert, J., Milligan, P.A., Rohou, S., Staab, A., Steimer, J.L., Tornøe, C. and Visser, S.A. (2016): Good Practices in Model-Informed Drug Discovery and Development: Practice, Application, and Documentation. CPT: Pharmacometrics Syst. Pharmacol., 5, 93-122.

Martin, F., Thomson, T.M., Sewer, A., Drubin, D.A., Mathis, C., Weisensee, D., Pratt, D., Hoeng, J. and Peitsch, M.C. (2012): Assessment of network perturbation amplitudes by applying high-throughput data to causal biological networks. BMC Syst. Biol., 6, 54 .

Morgan, S.J., Elangbam, C.S., Berens, S., Janovitz, E., Vitsky, A., Zabka, T., Conour, L. (2013): Use of animal models of human disease for nonclinical safety assessment of novel pharmaceuticals. Toxicol. Pathol., 41, 508-518.

Olson, H., Betton, G., Robinson, D., Thomas, K., Monro, A., Kolaja, G., Lilly, P., Sanders, J., Sipes, G., Bracken, W., Dorato, M., Van Deun, K., Smith, P., Berger, B. and Heller, A. (2000): Concordance of the toxicity of pharmaceuticals in humans and in animals. Regul. Toxicol. Pharmacol., 32, 56-67.

Rustici, G., Kolesnikov, N., Brandizi, M., Burdett, T., Dylag, M., Emam, I., Farne, A., Hastings, E., Ison, J., Keays, M., Kurbatova, N., Malone, J., Mani, R., Mupo, A., Pedro Pereira, R., Pilicheva, E., Rung, J., Sharma, A., Tang, Y.A., Ternent, T., 
Data-intensive Systems Biology/Toxicology/Pharmacology approaches

Tikhonov, A., Welter, D., Williams, E., Brazma, A., Parkinson, H. and Sarkans, U.. (2013): ArrayExpress update--trends in database growth and links to data analysis tools. Nucleic Acids Res., 41, D987-990.

Sahota, T., Danhof, M. and Della Pasqua, O. (2016): Pharmacology-based toxicity assessment: towards quantitative risk prediction in humans. Mutagenesis, 31, 359-374.

Su, Z., Fang, H., Hong, H., Shi, L., Zhang, W., Zhang, W., Zhang, Y., Dong, Z., Lancashire, L.J., Bessarabova, M., Yang, X., Ning, B., Gong, B., Meehan, J., Xu, J., Ge, W., Perkins, R., Fischer, M. and Tong, W. (2014): An investigation of biomarkers derived from legacy microarray data for their utility in the RNA-seq era. Genome Biol., 15, 523.

Subramanian, A., Tamayo, P., Mootha, V.K., Mukherjee, S., Ebert, B.L., Gillette, M.A., Paulovich, A., Pomeroy, S.L., Golub, T.R., Lander, E.S. and Mesirov, J.P. (2005): Gene set enrichment analysis: a knowledge-based approach for interpreting genome-wide expression profiles. Proc. Natl. Acad. Sci. USA, 102, 1554515550.

Uehara, T., Hirode, M., Ono, A., Kiyosawa, N., Omura, K., Shimizu, T., Mizukawa, Y., Miyagishima, T., Nagao, T. and Urushidani, T. (2008): A toxicogenomics approach for early assessment of potential non-genotoxic hepatocarcinogenicity of chemicals in rats. Toxicology, 250, 15-26.

Uehara, T., Ono, A., Maruyama, T., Kato, I., Yamada, H., Ohno, Y. and Urushidani, T. (2010): The Japanese toxicogenomics project: application of toxicogenomics. Mol. Nutr. Food Res., 54, 218227.

Vicini, P. and van der Graaf, P.H. (2013): Systems pharmacology for drug discovery and development: paradigm shift or flash in the pan? Clin. Pharmacol. Ther., 93, 379-381.

Wang, C., Gong, B., Bushel, P.R., Thierry-Mieg, J., Thierry-Mieg, D., Xu, J., Fang, H., Hong, H., Shen, J., Su, Z., Meehan, J., Li, X., Yang, L., Li, H., Łabaj, P.P., Kreil, D.P., Megherbi, D., Gaj, S., Caiment, F., van Delft, J., Kleinjans, J., Scherer, A., Devanarayan, V., Wang, J., Yang, Y., Qian, H.R., Lancashire,
L.J., Bessarabova, M., Nikolsky, Y., Furlanello, C., Chierici, M., Albanese, D., Jurman, G., Riccadonna, S., Filosi, M., Visintainer, R., Zhang, K.K., Li, J., Hsieh, J.H., Svoboda, D.L., Fuscoe, J.C., Deng, Y., Shi, L., Paules, R.S., Auerbach, S.S. and Tong, W. (2014): The concordance between RNA-seq and microarray data depends on chemical treatment and transcript abundance. Nat. Biotechnol., 32, 926-932.

Waters, M., Stasiewicz, S., Merrick, B.A., Tomer, K., Bushel, P., Paules, R., Stegman, N., Nehls, G., Yost, K.J., Johnson, C.H., Gustafson, S.F., Xirasagar, S., Xiao, N., Huang, C.C., Boyer, P., Chan, D.D., Pan, Q., Gong, H., Taylor, J., Choi, D., Rashid, A., Ahmed, A., Howle, R., Selkirk, J., Tennant, R. and Fostel, J. (2008): CEBS--Chemical Effects in Biological Systems: a public data repository integrating study design and toxicity data with microarray and proteomics data. Nucleic Acids Res., 36, D892900.

Webster, A.F., Zumbo, P., Fostel, J., Gandara, J., Hester, S.D., Recio, L., Williams, A., Wood, C.E., Yauk, C.L. and Mason, C.E. (2015): Mining the Archives: A Cross-Platform Analysis of Gene Expression Profiles in Archival Formalin-Fixed ParaffinEmbedded Tissues. Toxicol. Sci., 148, 460-472.

Xu, J., Gong, B., Wu, L., Thakkar, S., Hong, H. and Tong, W. (2016): Comprehensive Assessments of RNA-seq by the SEQC Consortium: FDA-Led Efforts Advance Precision Medicine. Pharmaceutics, $8,8$.

Xu, Y., Dai, Z., Chen, F., Gao, S., Pei, J. and Lai, L. (2015): Deep Learning for Drug-Induced Liver Injury. J. Chem. Inf. Model, 55, 2085-2093.

Yang, K., Woodhead, J.L., Watkins, P.B., Howell, B.A. and Brouwer, K.L. (2014): Systems pharmacology modeling predicts delayed presentation and species differences in bile acid-mediated troglitazone hepatotoxicity. Clin. Pharmacol. Ther., 96, 589598.

Yin, S. and Kaynak, O. (2015): Big Data for Modern Industry: Challenges and Trends. Proc. IEEE, 103, 143-146. 